\title{
Materialising Gender-fluidity through Fashion
}

As the final year comprehensive design project for the Honours Degree of Bachelor of Design, Department of Integrated Design, Faculty of Architecture, University of Moratuwa, I have selected a project which is based upon my own clothing brand. It is an emerging ready-to-wear clothing brand based in Sri Lanka, which was launched in August 2020 through the Colombo Fashion Week named "HARID". Currently, HARID retails at the Design Collective store in Colombo for a consumer group based upon it. The brand philosophy of HARID is to challenge gender-related stereotypical concepts. As the brand identity, HARID uses heritage craft practices.

As the next milestone of a Sri Lankan based clothing brand, I want to expand the brand for an international market to get a better global brand presence as well as to open global retailing opportunities.

According to the brand philosophy, I have considered "Gender-fluidity" as the next initiation for the concept. A Gender-fluid person could be either more masculine, more feminine, both masculine and feminine, or neither of them. That means their gender identity or gender expression shifts from time to time. When a person has more masculine gender expression or identity, that means that person has more masculine qualities compared to feminine qualities. Even though masculine and feminine qualities may differ from context to context, there are universal masculine and feminine qualities defined by the World Health Organization.

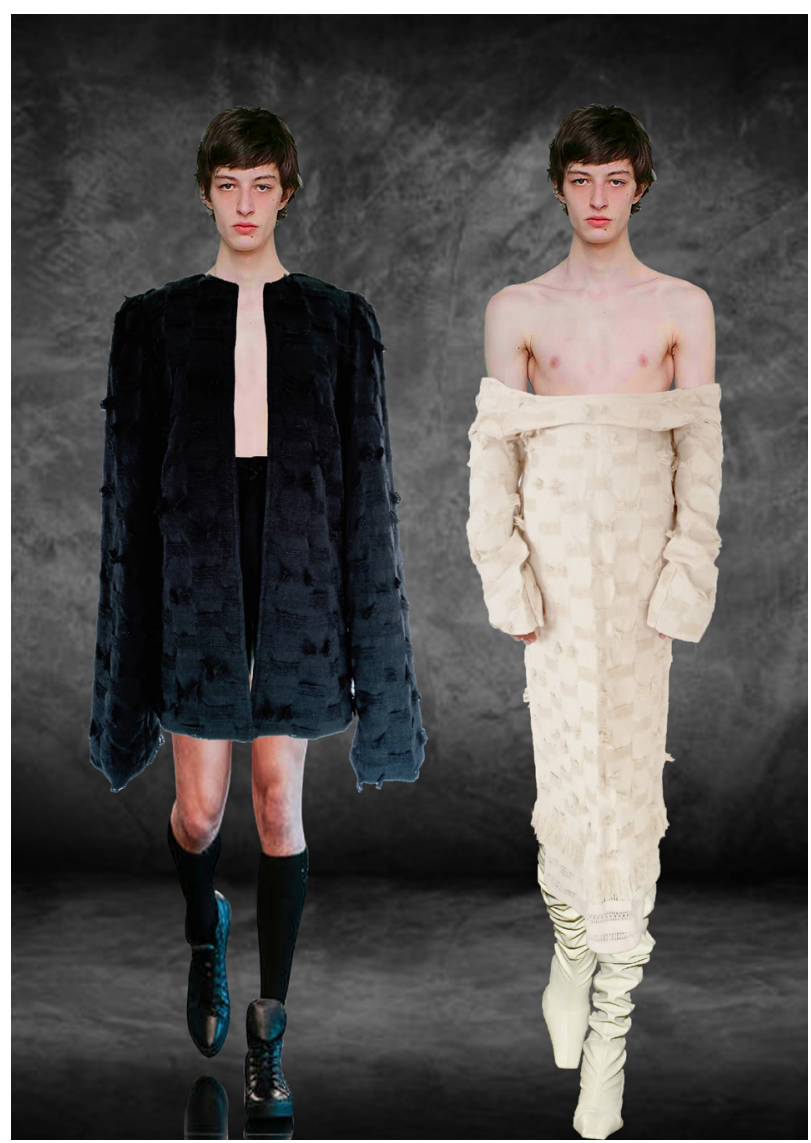

65 Even though

masculine and feminine qualities may differ

from context to context, there are universal masculine and feminine qualities defined by the World Health Organization 
Since my goal is to expand the brand into an international market, by using an online survey among the Facebook group which is "Gender-Fluid", I conducted an online survey and gathered geographic, demographic, and psychographic attributes of them. As further research, I interviewed a London based fashion designer "Eden Loweth" through Instagram. From the research study I was able to find out their overall silhouette preferences as well.

As a Sri Lankan based designer who is aiming to reach the international market, I wanted to incorporate Sri Lankan essence, and so that I researched about the philosophical background of Sri Lankan clothing. I was able to find out that Sri Lankan traditional attires, which are "Mulu aduma" and "Ohoriya", have been influenced by foreign culture, yet adopted according to suit the local context as dynamic and hybrid entities. Therefore, I have used that rationale in developing my collection.

After a comprehensive research, I have selected to expand the brand through London Fashion Week, since it has different platforms for upcoming designers and brands, supports gender-related concepts and can show off brand stories. London Fashion Week has different designer application categories. After personally contacting the British Fashion Council, which is the mother company of London Fashion Week, they suggested me to go through the designer application category, "Digital Profile" based on the status of my brand.

According to the brand identity, for this collection I have used local hand weaving as the heritage craft practice since, London Fashion Week also encourages designers to use ethical and locally manufactured materials and as well as I can promote it to the international audience with a contemporary approach. Therefore, I have materialized the initially identified masculine and feminine qualities by experimenting different types of local hand weaving techniques with different yarn types. With the initial experiments I have selected plain weaving, "pick-up motifs" technique and "gatapismenthu" technique to execute this collection.

As the colour palette, I have selected black and off white since, they are neutral and hence there

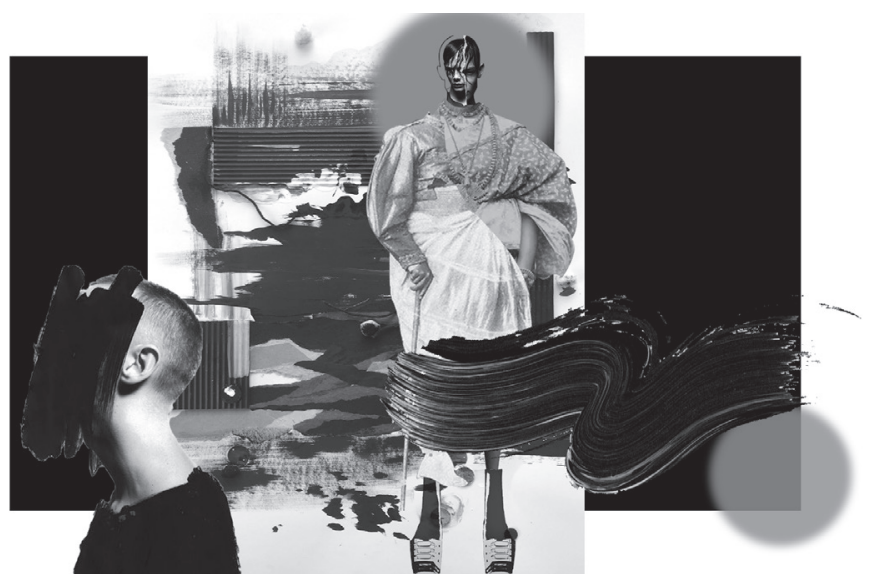

would not be any biasness towards either masculinity or femininity and as well as I can highlight the technique. This collection can be used as an inspiration to come up with more wearable clothing for people who identify themselves as Gender-fluid.

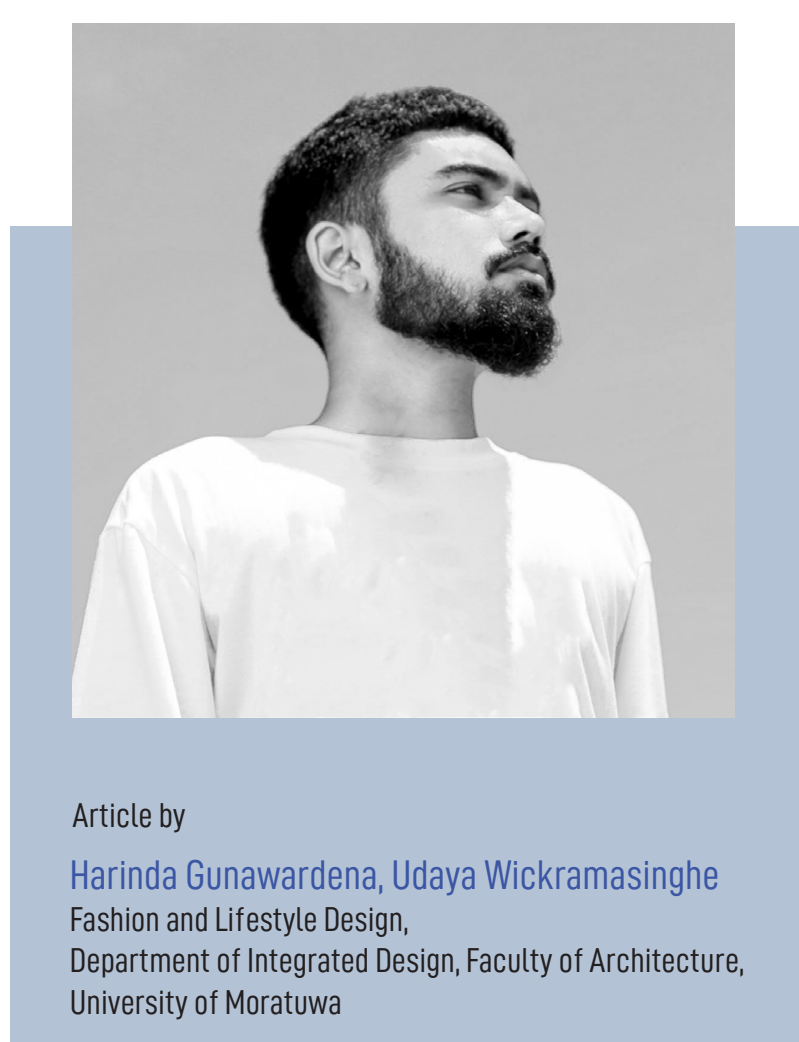

